\title{
Absolute phase estimation: adaptive local denoising and global unwrapping
}

\author{
Jose Bioucas-Dias, ${ }^{1, *}$ Vladimir Katkovnik, ${ }^{2,3}$ Jaakko Astola, ${ }^{2,4}$ and Karen Egiazarian ${ }^{2,5}$ \\ 'Instituto de Telecomunicações, Instituto Superior Técnico, TULisbon, 1049-001 Lisboa, Portugal \\ ${ }^{2}$ Signal Processing Institute, University of Technology of Tampere, P. O. Box 553, Tampere, Finland \\ ${ }^{3}$ katkov@cs.tut.fi \\ ${ }_{4 j t a @ c s . t u t . f i}$ \\ ${ }^{5}$ karen@cs.tut.fi \\ *Corresponding author: bioucas@|x.it.pt
}

Received 13 June 2008; revised 4 August 2008; accepted 6 August 2008; posted 4 September 2008 (Doc. ID 97398); published 7 October 2008

\begin{abstract}
The paper attacks absolute phase estimation with a two-step approach: the first step applies an adaptive local denoising scheme to the modulo- $2 \pi$ noisy phase; the second step applies a robust phase unwrapping algorithm to the denoised modulo- $2 \pi$ phase obtained in the first step. The adaptive local modulo- $2 \pi$ phase denoising is a new algorithm based on local polynomial approximations. The zero-order and the firstorder approximations of the phase are calculated in sliding windows of varying size. The zero-order approximation is used for pointwise adaptive window size selection, whereas the first-order approximation is used to filter the phase in the obtained windows. For phase unwrapping, we apply the recently introduced robust (in the sense of discontinuity preserving) PUMA unwrapping algorithm [IEEE Trans. Image Process. 16, 698 (2007)] to the denoised wrapped phase. Simulations give evidence that the proposed algorithm yields state-of-the-art performance, enabling strong noise attenuation while preserving image details. (C) 2008 Optical Society of America

OCIS codes: $\quad 100.5088,110.5086,280.0280$.
\end{abstract}

\section{Introduction}

Many remote sensing systems exploit phase coherence between transmitted and scattered waves to infer information about physical and geometric properties of illuminated objects, such as shape, deformation, movement, and structure of an object's surface. Phase estimation plays, therefore, a central role in these coherent imaging systems. For instance, in synthetic aperture radar interferometry (InSAR), the phase values are proportional to the terrain elevation height; in magnetic resonance imaging, the phase is used to measure temperature, to map the main magnetic field inhomogeneities, to identify veins in the tissues, and to segment water from

0003-6935/08/295358-12\$15.00/0

(C) 2008 Optical Society of America fat. Other examples can be found in adaptive optics, diffraction tomography, nondestructive testing of components, and deformation and vibration measurements (see, e.g., [1-6]).

In all these applications the observation mechanism is a $2 \pi$-periodic function of the true phase, hereafter termed absolute phase. The mapping of this function in the interval $[-\pi, \pi)$ yields the so-called principal phase values, or wrapped phases, or interferogram; if the true phase is outside the interval $[-\pi, \pi)$, the associated observed value is wrapped into it, corresponding to the addition/subtraction of an integer number of $2 \pi$. It is thus impossible to unambiguously reconstruct the absolute phase, unless additional assumptions are introduced into this inference problem.

Many approaches to absolute phase estimation follow a two-step procedure: in the first step, the 
wrapped phase is inferred from noisy wrapped observations; in the second step, the absolute phase is inferred from the wrapped estimate obtained in the first step. The latter procedure is known as phase unwrapping.

Owing to the periodic observation mechanism, phase unwrapping is a hard problem that has since long fostered active research. In fact, if the magnitude of phase variations between neighboring pixels is larger than $\pi$, i.e., the so-called Itoh condition [7] is violated, then the inference of the $2 \pi$ multiples is an ill-posed problem. These violations may be due to spatial undersampling, discontinuities, or noise. To deal with these difficulties, some sort of $a$ priori information has to be used. A classical approach implements path-dependent local techniques [8,9], where a pixel-by-pixel unwrapping is confirmed by local phase congruence tests. Work [10] exploits special wrapped regions where the phase have the same $2 \pi$ multiple. The algorithm is based on imitation of four phase-shifts in observations (by $\pi / 2$ ). The four different wrapped phases generated in this way are used for unwrapping. A more recent direction formulates the phase unwrapping as a regularized inverse problem, leading to path-independent optimization techniques [11-16] (see also [8] for a complete account of the path-following techniques and a partial account of global methods). In some applications, such as in InSAR, it is possible to infer maps indicating the quality of the observed phase in each pixel. Irrespective of the approach, the use of this external information eases, often significantly, the phase unwrapping problem.

The observation noise plaguing many coherent imaging systems introduces further difficulties in the phase reconstruction, as the phase unwrapping methods developed for noiseless data are very sensitive to noise. One of the first and natural ideas is prefiltering the noisy wrapped data and then using it for further processing, in particular for phase unwrapping. However, a phase fringe pattern (sets of points in the phase image domain for which the absolute phase takes values between two consecutive $2 \pi$ multiples) is a very delicate object with crucial details that are easily damaged in prefiltering. If the noise level is small, any reasonable filtering leads to acceptable results. However, as the noise level increases the standard approaches often damage data in such a way that further unwrapping becomes impossible.

Prefiltering the noisy wrapped phase for unwrapping is a difficult problem because of the nonadditive nature of the phase noise, undersampling (aliasing), and wrapping effects. Time-frequency analysis based on Fourier transform is a conventional tool in fringe analysis and fringe filtering. In particular, it has been demonstrated in [17] that a fringe pattern processed locally or block by block using the windowed Fourier transform method removes artifacts in phase measurements better than the simple carrier based Fourier transform methods such as the windowed Fourier ridges, the wavelet transform, and the regularized phase tracking. Presently, the windowed Fourier transform method is considered to be a promising tool among the spatial techniques in use for phase measurement [6].

At this point, we would like to emphasize that prefiltering, although desirable, is a rather delicate task. In fact, if prefiltering is too strong, the essential pattern of the absolute phase coded in the wrapped phase is damaged, and the reconstruction of absolute phase is compromised. On the other hand, if we do not filter, the unwrapping may be impossible because of the noise. A conclusion is, therefore, that filtering is crucial but should be designed very carefully. One of the ways to ensure efficiency is to adapt the strength of the prefiltering according to the phase surface smoothness and the noise level.

\section{A. Proposed Approach}

Here we propose a novel filtering technique based on local polynomial approximation with a varying adaptive neighborhood used in reconstruction [18]. We assume that the absolute phase is a piecewise smooth function, which is well approximated by a polynomial in a neighborhood of the estimation point. Besides the wrapped phase, the size and possibly the shape of this neighborhood are estimated. The technique is based on two independent ideas: local approximation for design of nonlinear filters (estimators) and adaptation of these filters to unknown smoothness of the spatially varying absolute phase. We use local polynomial approximation (LPA) for approximation in a sliding varying size window (Chap. 2 of [18]) and intersection of confidence intervals (ICI) for window size adaptation (Chap. 6 of [18]).

The adaptiveness introduced by ICI trades bias with variance in such a way that the window size stretches in areas where the underlying true phase is smooth and shrinks otherwise, namely in the presence of discontinuities. The phase unwrapping equipped with this adaptive LPA prefiltering yields very good accuracy of the phase reconstruction, quite often overcoming the state-of-the-art algorithms developed for noisy phase unwrap.

The polynomial modeling is a popular idea for both wrapped phase denoising and noisy phase unwrap. Using the local polynomial fit in terms of the phase tracking for the phase unwrap is proposed in [19], where it is exploited for the initialization of a global optimization procedure. The efficiency of the local fitting of the phase is demonstrated in [20], in particular for phase unwrapping for two-dimensional magnetic resonance imaging data. In [21] the linear local polynomial approximation of height profiles is used for the surface reconstruction from the multifrequency InSAR data.

Different modifications of the local polynomial approximation oriented to wrapped phase denoising are introduced in regularized phase-tracking [22,23], multiple-parameter least squares [24], and windowed Fourier ridges [17]. The comparative analysis 
of these methods produced in [25] shows that they yield comparable performance and that the differences are mainly due to calculations and algorithm implementations.

Compared with these works, the main novelty introduced in this paper is the window size selection adaptiveness introduced by the ICI technique, which locally adapts the amount of smoothing according to the data. In particular, the discontinuities are preserved, what is a sine qua non condition for the success of the posterior unwrapping; in fact, as discussed in [15], it is preferable to unwrap the noisy interferogram than a filtered version in which the discontinuities or the areas of high phase rate have been washed out.

The material herein presented is an elaboration of [26] and a development of the phase-tracking unwrap proposed in [27], where the ICI adaptive varying windows are used for the phase estimates calculated by recursive local minimization of the least square criterion. In this paper we are mainly focused on filtering the wrapped phase as the prefiltering procedure for the forthcoming unwrapping.

Experiments based on simulations and real data gives evidence that the developed filtering is very efficient. We come to this conclusion by comparing the true phase with the unwrapping obtained with PUMA [16] applied on the filtered wrapped phase. PUMA is able to preserve discontinuities by using graph cut based methods to solve the integer optimization problem associated with phase unwrapping. The reconstructed error is comparable to or better than state-of-the-art algorithms developed for noisy phase unwrapping, of which the $Z \pi M$ [15] and the PhaseLa [27] are two examples.

The paper is organized as follows. Section 2 introduces the zero and the first orders polynomial local approximations of the phase. Section $\underline{3}$ introduces the ICI concept. Section $\underline{4}$ presents the pseudocode of the overall algorithm which we have termed PEARLS (for phase estimation using adaptive regularization based on local smoothing). Finally, Section 5 presents a series of experiments with simulated data and a SAR data set generated from real data.

\section{Local Polynomial Phase Approximations}

The details of the observation models relating the noisy wrapped phase with the true phase depend on the coherent imaging system under consideration (see, e.g., $[8,15,27]$ for an account of observation models in different coherent imaging systems). Nevertheless, the essential of all these observation mechanisms is captured by the relation

$$
z=A \exp (j \varphi)+n, \quad A>0,
$$

where $n=n_{I}+j n_{Q}$ is complex-valued zero-mean circular white noise of variance $2 \sigma^{2}$ (i.e., $n_{I}$ and $n_{Q}$ are zero-mean independent Gaussian random variables with variance $\sigma^{2}$ ). Given that the noise is additive, we define the signal-to-noise-ratio as $\mathrm{SNR} \equiv 1 /\left(2 \sigma^{2}\right)$. Figure 1 illustrates the different components of the observed model in the complex plane: $\varphi$ is the true phase, $\phi$ is the observed phase, and $\phi_{n}$ is the phase component of $\phi$ due to noise vector $n$. The phase $\phi$ is given by

$$
\phi=\operatorname{angle}(z)=W\left(\varphi+\phi_{n}\right), \quad \phi \in[-\pi, \pi),
$$

where $W$ is the wrapping operator mapping the noisy phase $\varphi+\phi_{n}$ into the basic phase interval $[-\pi, \pi)$. For $\phi_{n}=0$, there is an obvious link between the wrapped $\phi$ and nonwrapped absolute phase $\varphi, \varphi=\phi+2 \pi k$, $\phi \in[-\pi, \pi)$, where $k$ is an integer. The basic unwrapping problem is to reconstruct $\varphi(x, y), x, y \in X \subset \mathbb{Z}^{2}$, from the observations $\phi(x, y)$. There is, of course, no one-to-one relation between the wrapped and the absolute phases.

Let us define the parameterized family of first-order polynomials:

$$
\tilde{\varphi}(u, v \mid \mathbf{c})=p^{T}(u, v) \mathbf{c},
$$

where $p=\left[p_{1}, p_{2}, p_{3}\right]^{T}=[1, u, v]^{T}$ and $\mathbf{c}=\left[c_{1}, c_{2}, c_{3}\right]^{T}$ is a vector of parameters. Assume that in some neighborhood of the point $(x, y)$, the phase $\varphi$ is well approximated by an element of the family (2); i.e., for $\left(x_{s}, y_{s}\right)$ in a neighborhood of the origin, there exists a vector $\mathbf{c}$ such that

$$
\varphi\left(x+x_{s}, y+x_{s}\right) \simeq \tilde{\varphi}\left(x_{s}, y_{s} \mid \mathbf{c}\right)
$$

To infer this vector, we compute

$$
\hat{\mathbf{c}}=\arg \min _{\mathbf{c}} L_{h}(\mathbf{c}),
$$

where $L_{h}(\mathbf{c})$ is a measure of the data misfit with respect to $z_{\phi} \equiv z /|z|=e^{j \phi}$, defined as

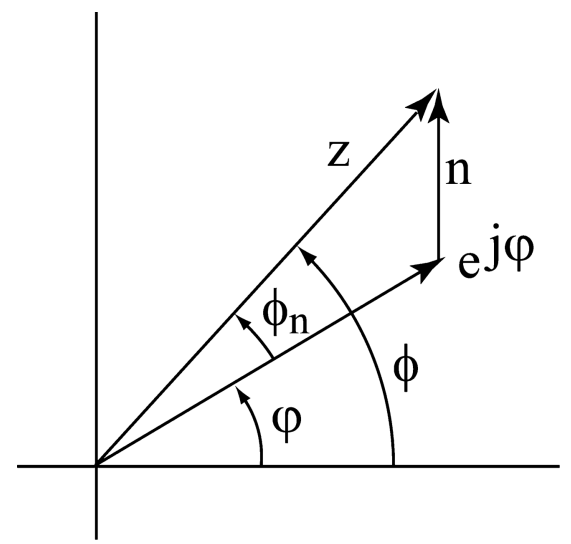

Fig. 1. Illustration of the observed phase model (1): $\varphi$ is the true phase, $\phi$ is the observed phase, and $\phi_{n}$ is the phase component of $\phi$ due to noise vector $n$ 


$$
\begin{aligned}
L_{h}(\mathbf{c})= & \frac{1}{2} \sum_{s} w_{h, s} \mid z_{\phi}\left(x+x_{s}, y+y_{s}\right) \\
& -\exp \left(\left.\tilde{j} \tilde{\varphi}\left(x_{s}, y_{s} \mid \mathbf{c}\right)\right|^{2}\right. \\
= & \sum_{s} w_{h, s}\{1 \\
& \left.-\cos \left[\phi\left(x+x_{s}, y+y_{s}\right)-\tilde{\varphi}\left(x_{s}, y_{s} \mid \mathbf{c}\right)\right]\right\},
\end{aligned}
$$

where $w_{h, s}$ are window weights parameterized by $h$, for $h \in H \equiv\left\{h_{1}<h_{2}<\ldots<h_{J}\right\}$, and $s$ is a window relative index. It can be verified by routine calculations that, for the considered linear phase model, the optimal solution $\hat{\mathbf{c}}$ is given by (see, e.g., Chap. 6 of [28])

$$
\begin{gathered}
\left(\hat{c}_{2}, \hat{c}_{3}\right) \in \arg \max _{c_{2}, c_{3}}\left|F_{h}\left(c_{2}, c_{3}\right)\right|, \\
\hat{c}_{1}=\operatorname{angle} F_{h}\left(\hat{c}_{2}, \hat{c}_{3}\right),
\end{gathered}
$$

where $F_{h}\left(c_{2}, c_{3}\right)$ is the windowed discrete Fourier transform of the normalized data $z_{\phi}=z /|z|$ at point $(x, y)$ and frequency $\left(c_{2}, c_{3}\right)$, i.e.,

$$
F_{h}\left(c_{2}, c_{3}\right)=\sum_{s} w_{h, s} z_{\phi}\left(x+x_{s}, y+y_{s}\right) e^{-j\left(c_{2} x_{s}+c_{3} y_{s}\right)} .
$$

Then, the phase estimate $\hat{c}_{1}$ is the argument (angle) of the complex-valued discrete Fourier transform $F_{h}$ calculated at the point where the maximum value of the Fourier spectrum is achieved. Equations (4) and (5) show that the standard fast Fourier transform $(\overline{\mathrm{F}} \mathrm{FT})$ algorithm can be used to efficiently compute the vector $\left(c_{1}, c_{2}, c_{3}\right)$.

According to the model in Eq. (2) and the hypothesis in Eq. (3), we have $\varphi(x, y)=\tilde{\varphi}(0,0 \mid \mathbf{c})=c_{1}$. Since we just have an estimate of $c_{1}$, we write

$$
\hat{\varphi}_{h}(x, y)=\hat{c}_{1}(x, y),
$$

where argument $(x, y)$ and index $h$ stress, respectively, the local nature of the phase estimate and its dependency on window size $h$.

We wish to emphasize the nonparametric nature of the introduced estimator. Indeed, we start with the parametric model in Eq. (2) linear on $x$ and $y$ and could expect that the approximation (estimate) is also linear on $x$ and $y$. However, the fit is used in the polynomial approximation in Eq. (2) only for one central point, $x_{s}=y_{s}=0$. The result of this pointwise use of the approximation is that the parametric estimate in Eq. (2) becomes nonparametric, with $\hat{\varphi}_{h}(x, y)$ depending in a nonlinear way on $x$ and $y$ [18]. All ideas of the standard LPA concerning window $w$ (shape, anisotropy, directionality, etc.), scaling $h$ (scalar, multivariate), and the estimation of the signal and derivatives are naturally valid in this nonparametric pointwise estimation.

By setting $c_{2}=0$ and $c_{3}=0$ in Eq. (2), a minor modification, the calculus carried out for the first- order polynomial approximation leads to the zeroorder polynomial based estimate:

$$
\hat{\varphi}_{h}(x, y)=\hat{c}_{1}(x, y)=\operatorname{angle}\left[F_{h}(0,0)\right] .
$$

To characterize, very briefly, the zero-order and the first-order phase approximations, let us assume that observed data is exactly modeled, within a given window, by a first-order polynomial of parameter vector $\mathbf{c}^{\prime}=\left(c_{1}^{\prime}, c_{2}^{\prime}, c_{3}^{\prime}\right)$. We have then $z_{\phi}\left(x+x_{s}, y+y_{s}\right)=$ $e^{j\left(c_{1}^{\prime}+c_{2}^{\prime} x_{s}+c_{3}^{2} y_{s}\right)}$ and

$$
\begin{aligned}
F_{h}\left(c_{2}, c_{3}\right) & =\sum_{s} w_{h, s} z_{\phi}\left(x+x_{s}, y+y_{s}\right) e^{-j\left(c_{2} x_{s}+c_{3} y_{s}\right)} \\
& =e^{j c_{1}^{\prime}} \sum_{s} w_{h, s} e^{-j\left[\left(c_{2}-c_{2}^{\prime}\right) x_{s}+\left(c_{3}-c_{3}^{\prime}\right) y_{s}\right]} \\
& \left.=e^{j c_{1}^{\prime}} W_{h}\left[\left(c_{2}-c_{2}^{\prime}\right) x_{s}+\left(c_{3}-c_{3}^{\prime}\right) y_{s}\right)\right],
\end{aligned}
$$

where $W_{h}\left[\left(c_{2}-c_{2}^{\prime}\right) x_{s}+\left(c_{3}-c_{3}^{\prime}\right) y_{s}\right]$ is the Fourier transform of the window weights $w_{h, s}$. Assuming that $w_{h, s}$ are nonnegative, the maximum of $\left|W_{s}\left(c_{2}, c_{3}\right)\right|$ happens for $\left(c_{2}=0, c_{3}=0\right)$ and then we have the following approximations:

a. Zero-order approximation $\left(c_{2} \equiv 0\right.$ and $\left.c_{3} \equiv 0\right)$

$$
\hat{\varphi}_{h}(x, y)=\operatorname{angle}\left[F_{h}(0,0)\right]=c_{1}^{\prime}+\operatorname{angle}\left[W_{h}\left(-c_{2}^{\prime},-c_{3}^{\prime}\right)\right] ;
$$

\section{b. First-order approximation}

$$
\hat{\varphi}_{h}(x, y)=\operatorname{angle}\left[F_{h}\left(\hat{c}_{2}, \hat{c}_{3}\right)\right]=c_{1}^{\prime} .
$$

We conclude, therefore, that the zero-order approximation produces biased estimates, whereas the firstorder does not. However, if the window is symmetric, $W_{h}$ is real and positive for frequencies close to the origin. For these frequencies, the zero-order approximation is, thus, unbiased. According to the scaling property of the Fourier transform, increasing window spreads in the spatial domain corresponds to decreasing spreads of the respective Fourier transform. We conclude, therefore, that the zero-order approximation based on symmetric windows tends to yield unbiased wrapped phase estimates, at least for low window sizes and low frequencies (phase slopes) $c_{2}$ and $c_{3}$.

Concerning computational complexity, the zeroorder approximation is much lighter than the firstorder approximation. Furthermore, as seen in Section $\underline{3}$, the inference of the best window size at a given point $(x, y)$ implies the determination of a series of wrapped phase estimates corresponding to an equal number of windows with different sizes. Aiming at a balance between computational complexity and quality of the estimates, we adopt the zero-order approximation to infer the window sizes and the first-order approximation to compute the wrapped phase estimates on the windows determined by the zero-order approximation. 


\section{Estimate Accuracy and Adaptation}

Given a two-dimensional symmetric window function $w$ and a window size (scale) parameter $h>0$, we define the scaled window $w_{h, s} \equiv w\left(x_{s} / h, y_{s} / h\right)$, centered at $(x, y)$. In particular, if we take the square uniform window $w=1$ for $|x| \leq 1$ and $|y| \leq 1$, and $w=$ 0 otherwise, we have $w_{h}=1$ for $|x| \leq h$ and $|y| \leq h$, and $w_{h}=0$ otherwise. A smaller or larger $h$ narrows or widens window $w_{h}$, respectively.

It is shown in Proposition 2 of [27] that, asymptotically for small noise level and small $h$, the phase estimate $\hat{\varphi}_{h}(x, y)=\hat{c}_{1}(x, y)$ given in Eq. ( $\left.\underline{6}\right)$ is unbiased with the variance:

$$
\sigma_{h}^{2}=\sigma^{2} \frac{\sum_{s} w_{h, s}^{2}}{\left(\sum_{s} w_{h, s}\right)^{2}} .
$$

This result is used for the adaptive selection of the window size. Let $\hat{\varphi}_{h}(x, y)$, for $h \in H$, be the respective phase estimates. The ICI rule is exploited to select the best window size. Given the estimates $\hat{\varphi}_{h}(x, y)$ and the respective variance, for $h \in H$, the confidence intervals of these estimates are defined as

$$
Q_{h}=\left\{\hat{\varphi}_{h}-\Gamma \cdot \sigma_{h}, \hat{\varphi}_{h}+\Gamma \cdot \sigma_{h}\right\},
$$

where $\Gamma>0$ is a parameter of the algorithm, and $\sigma_{h}$ is calculated according to Eq. (8).

The ICI rule defines the adaptive window size, denoted by $h^{+}$, as the largest $h \in H$ for which the estimate $\hat{\varphi}_{h}$ does not differ significantly from the estimates corresponding to the smaller window sizes. To identify this adaptive $h^{+}$, the successive intersection of the confidence intervals $Q_{h}$ is considered starting from $Q_{h_{1}}$ and $Q_{h_{2}}$. Specifically, the pairwise intersection of the intervals $Q_{h_{i}}, 1 \leq h_{j} \leq h_{i}$, is considered with increasing $h_{i}$. Let $h^{+}$be the largest of those $h_{i}$ for which the intervals $Q_{h_{i}}, 1 \leq h_{j} \leq h_{i}$, have a point in common. This $h^{+}$defines the adaptive window size and the adaptive estimate as $\hat{\varphi}_{h^{+}}$.

For the varying pointwise adaptive estimation, these calculations are produced for all points (pixels). In implementation, the ICI algorithm is used when the estimates for all points $(x, y)$ are already calculated for all $h$. Then the algorithm works as a selector of the proper window size estimate for each point from a given set of the estimates for all window sizes (Chap. 6 of [18]).

Parameter $\bar{\Gamma}$ in Eq. (9) controls the bias-variance balance in the estimate. Decreasing $\Gamma$ means a shift of this balance in favor of the bias, as smaller $\Gamma$ results in smaller bias of the estimate. On the contrary, increasing $\Gamma$ means a shift in favor of the variance, as larger $\Gamma$ results in smaller variance of the estimate but possible larger bias.

\section{PEARLS Algorithm}

We name the proposed algorithm the phase estimation using adaptive regularization based on local smoothing (PEARLS). The pseudocode for this algorithm is as follows:

\section{For every pixel $(x, y) \in X$ and $h \in H$ :}

a. Calculate the zero-order phase estimate according to Eq. (7), i.e.,

$$
\hat{\varphi}_{h}(x, y)=\operatorname{angle}\left[\sum_{s} w_{h, s} z_{\phi}\left(x+x_{s}, y+y_{s}\right)\right] ;
$$

b. Apply the ICI rule to the estimates $\hat{\varphi}_{h}(x, y)$ for the selection of the best window size $h^{+}(x, y)$;

c. Using Eq. (5) with $h=h^{+}(x, y)$, calculate $\hat{\varphi}_{h^{+}}$, the first-order phase estimates with adaptive window size;

End for

2. Unwrap phase $\hat{\varphi}_{h^{+}}$using the PUMA algorithm [16].

PEARLS computational complexity, measured in number of floating points operations, is dominated by steps $1 \mathrm{c}$ and 2 . Step 1c computes the vector $\left(\hat{c}_{1}, \hat{c}_{2}, \hat{c}_{3}\right)$ for every pixel in the image according to Eqs. (4) and (5). These estimates are efficiently determined by means of a zero-padded FFT. For FFTs of size $L^{2}$, the leading term of the computational complexity of step 1c is, thus, $\left(2 L^{2} \log _{2} L\right) n$, where $n$ is the number of image pixels.

Step 2, the PUMA phase unwrapping algorithm, has complexity $O\left(n^{2.5}\right)$, This is, however, the worst case figure. The practical complexity is very close to $O(n)$ [16]. Therefore, the overall complexity of PEARLS is $O(n)$. In practice, we have observed that $\left(2 L^{2} \log _{2} L\right) n$ is a good approximation of the time the algorithm takes. Finally, we note that step 1c can be parallelized and its time divided, approximately, by the number of processors used to compute the FFTs.

\section{Experimental Results}

Here we present several experiments illustrating the PEARLS competitiveness. For the phase unwrap of the filtered wrapped phase we use the PUMA algorithm [16], which is able to work with discontinuities. In what follows, LPA is exploited with the uniform square windows $w_{h}$ defined on the integer symmetric grid $\{(x, y):|x|,|y| \leq h\}$; thus, the number of pixels of $w_{h}$ is $(2 h+1)$. The ICI parameter was set to $\Gamma=2.0$, the window sizes to $H=\{1,2,3,4\}$, and the zeropadded FFTs sizes to $L \times L=64 \times 64$.

\section{A. Continuous Phase Surfaces}

\section{Gaussian Surface}

In this experiment we consider continuous phase surface. Here the word continuous is to be understood in the sense that there are no large walks of neighboring pairs of pixels with phase differences larger than $\pi$ in magnitude. 
Figure 2 shows estimation results for the Gaussian shaped surface $\varphi(x, y)=A_{\varphi} \exp \left[-x^{2} /\left(2 \sigma_{x}^{2}\right)-y^{2} /\left(2 \sigma_{y}^{2}\right)\right]$ with $\sigma_{x}=10, \sigma_{y}=15$, and $A_{\varphi}=14 \pi$. The maximum value of $\varphi$ is $14 \pi$ and the maximum values of the first differences are about $2.5 \mathrm{rad}$. With such high phase differences, even a noise of small variance leads to a difficult unwrapping problem due to many phase differences larger than $\pi$. The noisy observations were generated according to Eq. (1), for $A=1$, on the square grid with integer arguments $x, y,-49 \leq x$, $y \leq 50$. The noise standard deviation is set to $\sigma=$ 0.5 corresponding to $\mathrm{SNR}=3 \mathrm{~dB}$. In spite of this low value of SNR, PEARLS leads to good estimates. The improvement in the SNR (ISNR) of the wrapped estimate [see Fig. 2(c)], which we define as

$$
\mathrm{ISNR}=10 \log 10 \frac{\left\|e^{j \phi}-e^{j \varphi}\right\|^{2}}{\left\|e^{\widehat{j}_{h^{+}}}-e^{j \varphi}\right\|^{2}},
$$

is $\operatorname{ISNR}=10.8 \mathrm{~dB}$. The root mean square error (RMSE) of the absolute phase estimate displayed in Fig. 2(d), is RMSE $=0.15 \mathrm{rad}$. Notice the ICI ability to locally adapt the amount of smoothness: the larger windows are selected in areas where a firstorder polynomial is a good approximation to the data and vice versa [see Fig. 2(b)].

Table 1 shows the RMSE values obtained with the PEARLS algorithm, as a function of the observation noise standard deviation $\sigma$. For comparison purposes, we have also computed the RMSE obtained with the PhaseLa [27] and $Z \pi M$ [15] algorithms, which implement filtering plus unwrapping. The unwrapping based on the PEARLS denoising yields consistently comparable or better performance than PhaseLa and $Z \pi M$ algorithms. The advantage increases for large values of $\sigma$, corresponding to the more challenging scenarios.

The last column of Table 1 displays the times that each algorithm takes in a $\overline{\mathrm{PC}}$ equipped with a Pentium 4 running at $3.4 \mathrm{GHz}$. There is just one column as the times depend very little on the noise variances. These times should be understood only as indicative, because, although all the algorithms have been coded

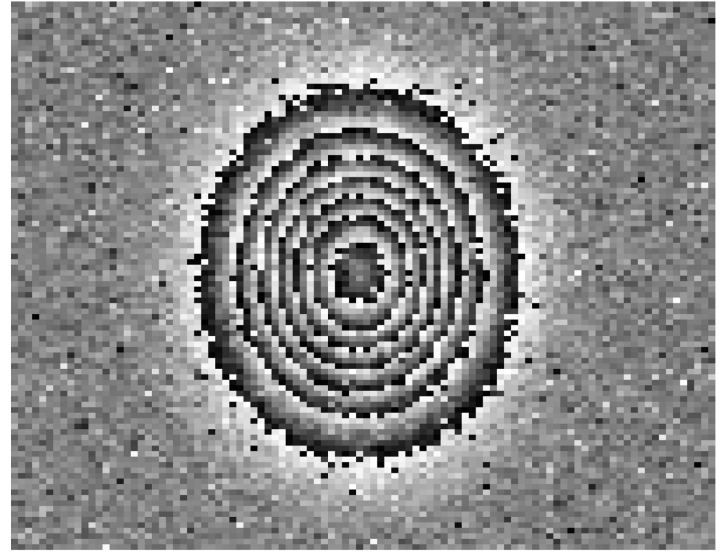

(a) Wrapped noisy phase.

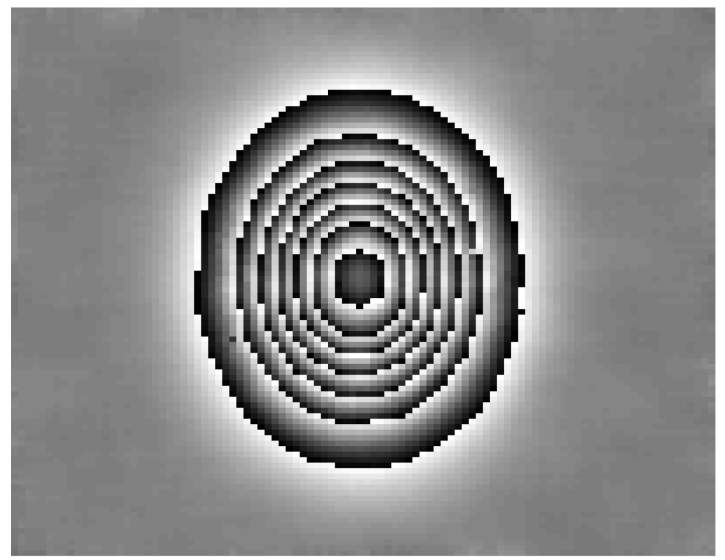

(c) wrapped denoised Phase

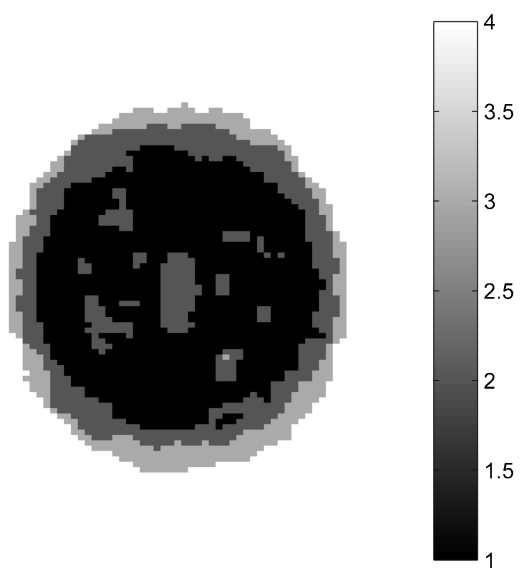

(b) Window parameter $h$ given by ICI.

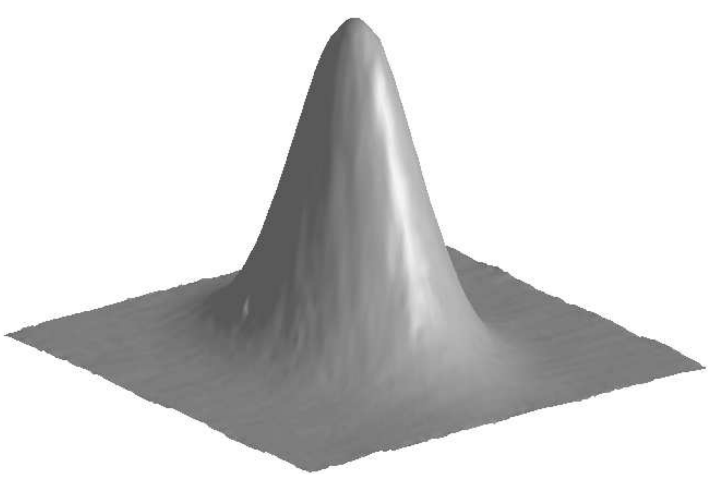

(d) Absolute phase estimate.

Fig. 2. PEARLS estimation results for a Gaussian shaped surface with $\sigma=0.5$ corresponding to $\mathrm{SNR}=3 \mathrm{~dB}$. The window size parameter $h$ shown in part (b) illustrates the ICI ability to locally adapt the amount of smoothness: the larger windows are selected in areas were a first-order polynomial is a good approximation to the data and vice versa. The denoised wrapped phase, shown in part (c) is clearly cleaner; the SNR improvement is of $10.8 \mathrm{~dB}$. The error of the reconstructed image in part (d) is RMSE $=0.15$ rad. 
Table 1. RMSE (in rad.) for PEARLS, PhaseLa, and $Z \pi M$ Algorithms

\begin{tabular}{lcccccc}
\hline Algorithm $\backslash \sigma$ & 0.75 & 0.5 & 0.25 & 0.05 & 0.01 & Time (s) \\
PEARLS & 0.34 & 0.15 & 0.09 & 0.05 & 0.03 & 30 \\
PhaseLa & fail & 0.18 & 0.11 & 0.04 & 0.03 & 24 \\
$Z \pi M$ & fail & 0.21 & 0.12 & 0.19 & 0.08 & 13 \\
\hline
\end{tabular}

in MATLAB, parts of $Z \pi M$ and PEARLS are implemented as $\mathrm{C}$ functions.

\section{Random Elevation Surface}

Figure 3(a), shows a noisy wrapped image corresponding to a random surface resulting from lowpass filtering an image of independent and identically distributed Gaussian noise. The additive noise level is $\mathrm{SNR}=3 \mathrm{~dB}$ and the magnitude of the first order differences is larger than $\pi$ in many areas of the image. The low SNR and high phase renders a hard absolute phase estimation problem. Figure 3(b) shows the size of the estimation windows. Again notice the ICI ability to locally adapt the amount of smoothness. Figure 3(c), shows that the estimate yielded by PEARLS is much cleaner than the original noisy wrapped image, corresponding to an improvement of ISNR $=4.8 \mathrm{~dB}$. Figure $3(\mathrm{~d})$ shows the reconstructed image by PUMA, corresponding to an $\mathrm{RMSE}=0.33 \mathrm{rad}$.

\section{B. Discontinuous Phase Surfaces}

\section{Clipped Gaussian}

In this experiment we illustrate the potential in handling discontinuities of bringing together the adaptive denoising and the unwrapping provided by LPA-ICI and PUMA, respectively. Figure 4 illustrates the performance of the algorithm in the Gaussian surface shown in Fig. 2 with one quarter set to zero. The noise standard deviation is set to $\sigma=0.5$, corresponding to $\mathrm{SNR}=3 \mathrm{~dB}$. The denoised wrapped phase shown in Fig. 4(c) is a clear improvement of the noisy version presented in Fig. 4(a). The strength of the smoothness shown in Fig. $\overline{4(\mathrm{~b})}$, imposed by the window sizes, is larger in areas where the absolute phase is smooth and smaller otherwise. In this way, the phase information in the neighborhood of discontinuities is not washed out, allowing PUMA

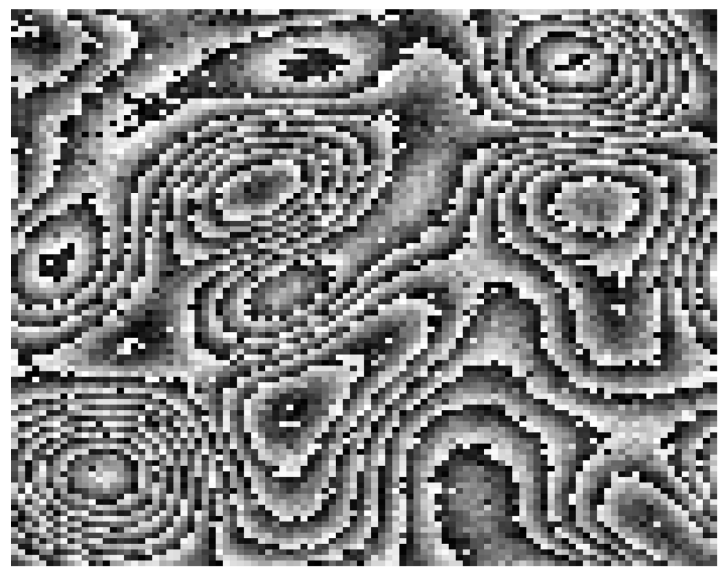

(a) Wrapped noisy phase.

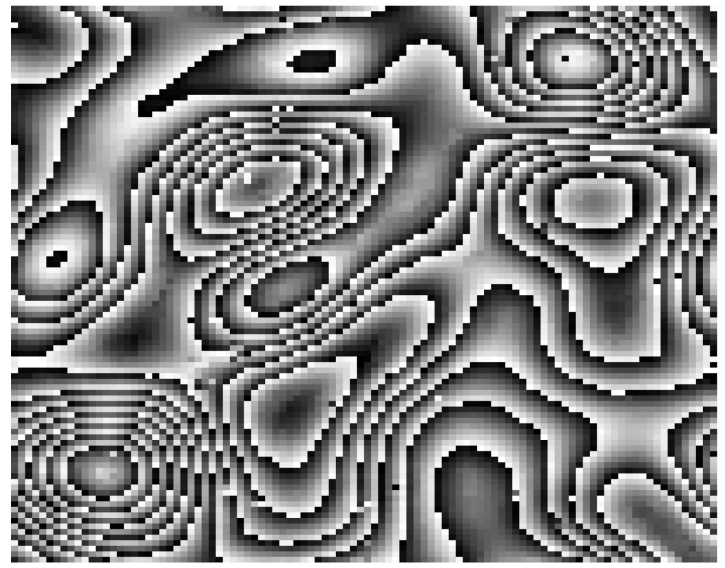

(c) Wrapped denoised Phase

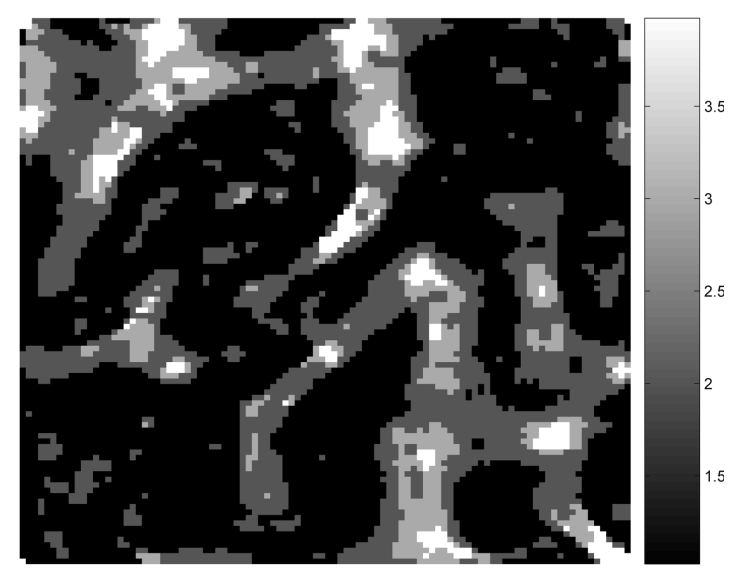

(b) Window size parameter $h$ given by ICI.

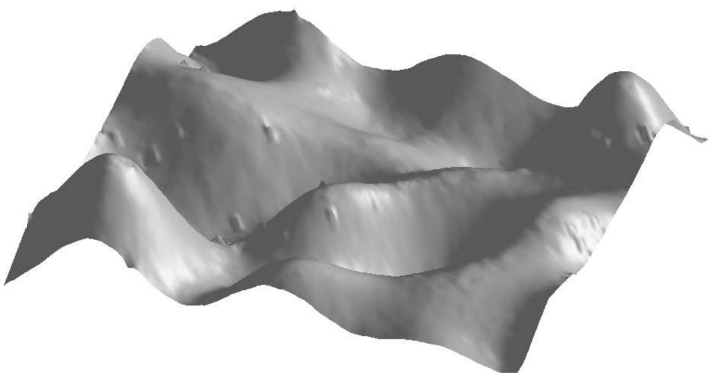

(d) Absolute phase estimate.

Fig. 3. As in Fig. 2, for the random surface shown in part (a), the denoised wrapped phase, shown in part (c), is clearly cleaner; the SNR improvement is ISNR $=4.8 \mathrm{~dB}$. The error of the reconstructed image in part (d) is $\mathrm{RMSE}=0.33 \mathrm{rad}$. 


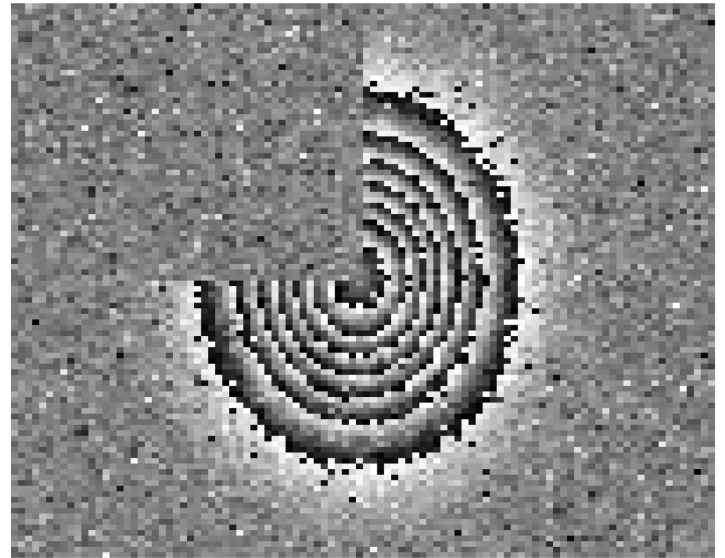

(a) Wrapped noisy phase.

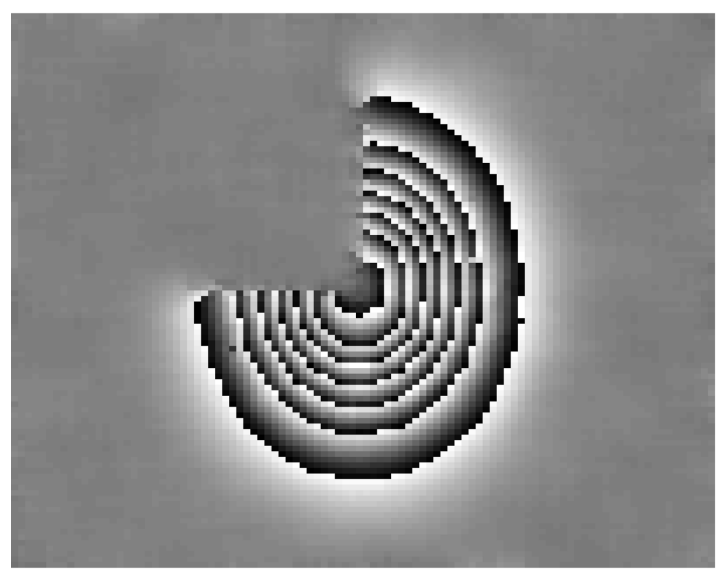

(c) Wrapped denoised phase.

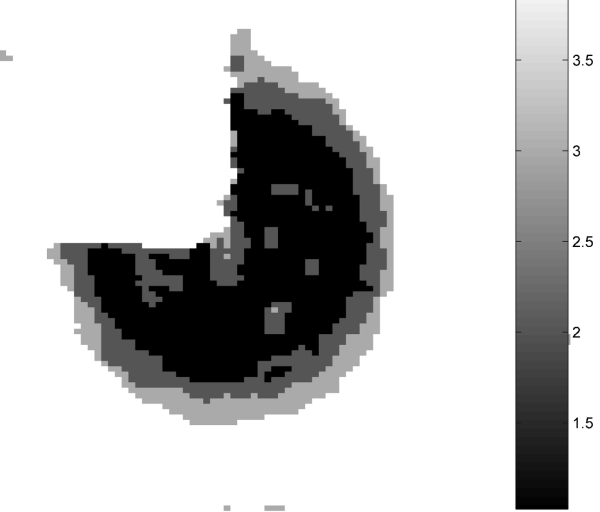

(b) Window size parameter $h$ given by ICI.

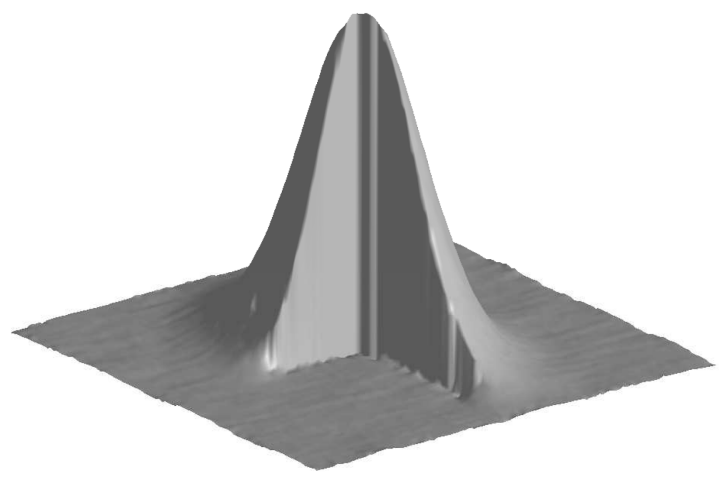

(d) Absolute phase estimate.

Fig. 4. As in Fig. 2, for the Gaussian shaped surface with a quarter set to zero shown in part (a), the noise variance is set to $\sigma=0.5$ corresponding to $\mathrm{S} \overline{\mathrm{N}} \mathrm{R}=3 \mathrm{~dB}$.

to produce an acceptable reconstruction. PhaseLa and $Z \pi M$ were not compared in this experiment because they are not conceived to blindly deal with discontinuities.

To further assess the added value of bringing together LPA-ICI and PUMA, we ran PUMA with and without prefiltering, for the noise standard deviation of $\sigma=0.5$ and $\sigma=0.75$, corresponding to $\mathrm{SNR}=3$ and $-0.5 \mathrm{~dB}$, respectively. The results are presented in Fig. 5. The denoising step obviously improves the reconstruction for the smooth areas where the larger values of the window sizes are used in the PEARLS algorithm. This improvement is overwhelming for the noise level $\sigma=0.75$, where the unwrapped nosy phase is totally useless.

\section{Simulated SAR Data Based on a Real Surface with Quality Maps}

Here we use a simulated InSAR example supplied in [8]. The data set was generated based on a real digital elevation model of mountainous terrain around Longs Peak, Colorado, using a high-fidelity InSAR simulator that models the SAR point spread function, the InSAR geometry, the speckle noise, and the layover and shadow phenomena. For a detailed description of the simulator see Chap. 3 of [8] and the references therein. Figure $\underline{6}$ shows a contour plot of the terrain used to generate the InSAR data. The size of the image in pixels is 458 (azimuth) $x$ 152 (range), corresponding to 1.7 and $1.1 \mathrm{~km}$, respectively. To compare the estimated surfaces directly with the ground truth, the surface has been resampled in the SAR slant plane.

Figure 7(a), shows the wrapped noisy phase. This image, distributed with [8], is the maximum likelihood estimate of the wrapped phase in windows of $4 \times 4$ assuming that the parameters of the SAR observation density are constant in these windows. The two flat regions in gray on the top and on the bottom of Fig. 7(a) correspond to undefined data due to the projection of the high terrain relief into the slant plane. The SAR layover phenomenon leads to very close fringes in some regions of the interferogram, clearly visible in the figure. In these sites, let $X_{0}$ denote this set of pixels, the assumption of constant phase within small windows is far from being true, leading to incorrect estimates of the principal phase 


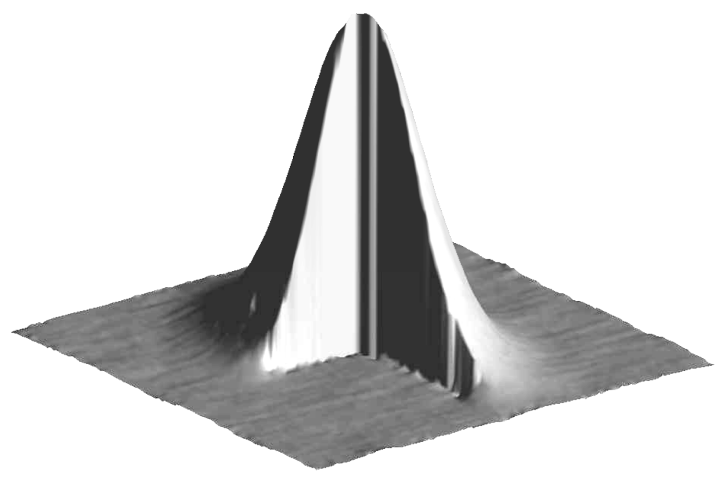

(a) PEARLS estimate $(\sigma=0.5)$.

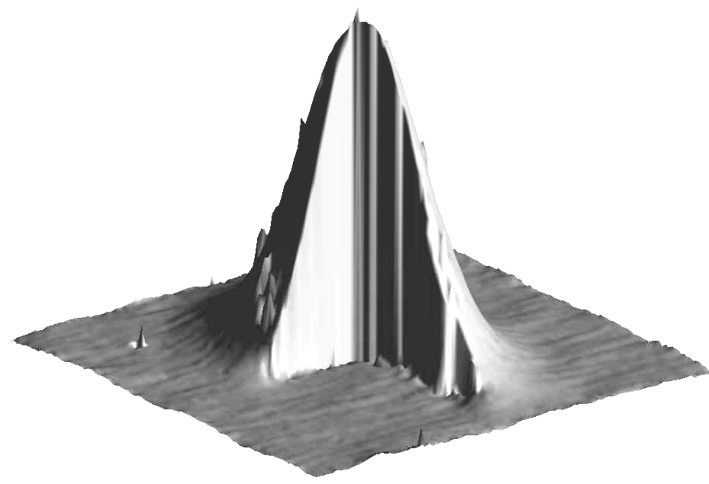

(c) PEARLS estimate $(\sigma=0.75)$.

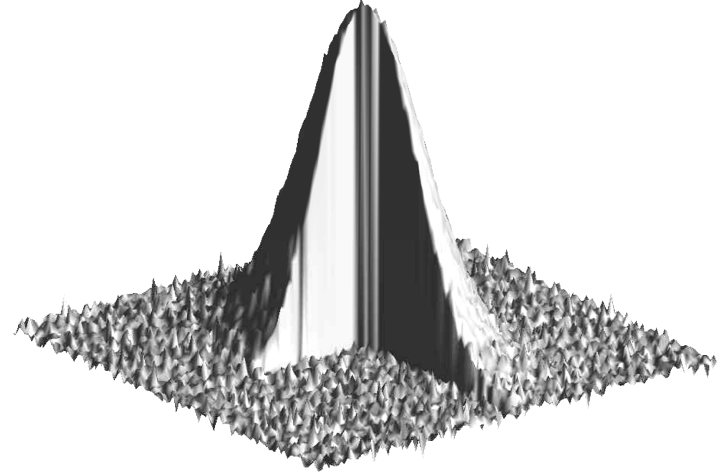

(b) PUMA estimate $(\sigma=0.5)$.

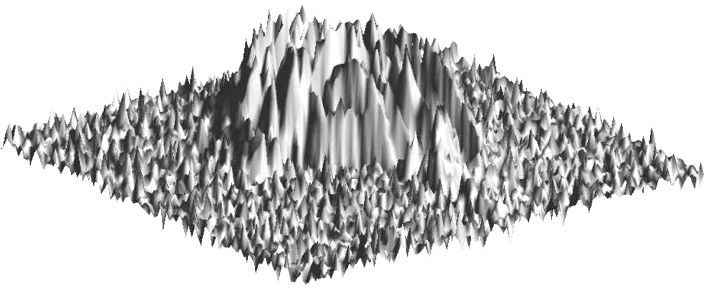

(d) PUMA estimate $(\sigma=0.75)$.

Fig. 5. Impact of denoising on PUMA unwrapping.

values in the SAR layover regions. Therefore, the wrapped phase in these regions is not used, as it is inconsistent with the true phase. In terms of the PEARLS algorithm, this means that the phase should be inferred from the data observed in the sub-

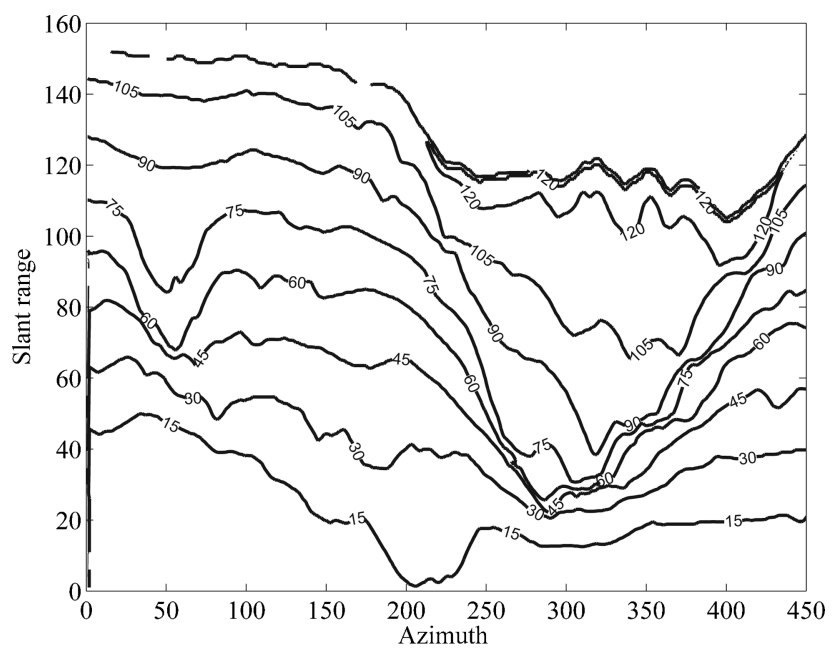

Fig. 6. Contour map (rad) of the terrain used to generate the InSAR data. The surface, a digital terrain elevation model of mountainous terrain around Longs Peak, Colorodo has been resampled in the SAR slant plane; it can be therefore directly compared with the estimated surfaces. (Data distributed with [8]). set $X-X_{0}$. To determine the set $X_{0}$, we have adopted the phase derivative and thresholding procedures presented in Chap. 3 of [8]. Figure 8 shows this set. Notice that the pixels in black are mostly in the layover regions.

Figure 7(b), shows the size of estimated windows by LPA-ICI. As in the previous examples, the LPA-ICI adaption mechanism has selected the smaller windows in the areas of high phase variability and vice versa. The denoised phase shown in Fig. 7 (c), is cleaner than the noisy version; nevertheless, its details are preserved. The improvement obtained in the set of pixels $X-X_{0}$ was ISNR $=4 \mathrm{~dB}$. Figure 7 (d), shows the histogram of the estimation error in this set, corresponding to an RMSE $=0.2 \mathrm{rad}$. The number of errors larger than $\pi$ is just 23. If we do not take these pixels into account, the error decreases to $\mathrm{RMSE}=0.17 \mathrm{rad}$.

For comparison purposes, we have run $Z \pi M$ on the same data set and in the same conditions. The results obtained, in line with those published in [15], are ISNR $=2 \mathrm{~dB}$ and $\mathrm{RMSE}=0.24 \mathrm{rad}$. The number of outliers is 27 and the error without these outliers is RMSE $=0.2 \mathrm{rad}$. PEARLS yields a better result owing to the adaptive window size scheme it implements.

Finally, Fig. 9 shows the true phase surface and the PEARL estimate. The values for the set $X_{0}$ have been 


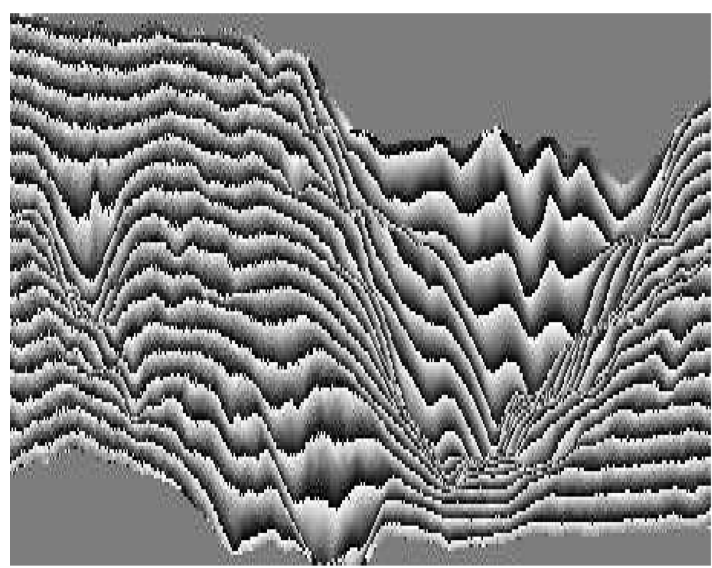

(a) Wrapped noisy phase.

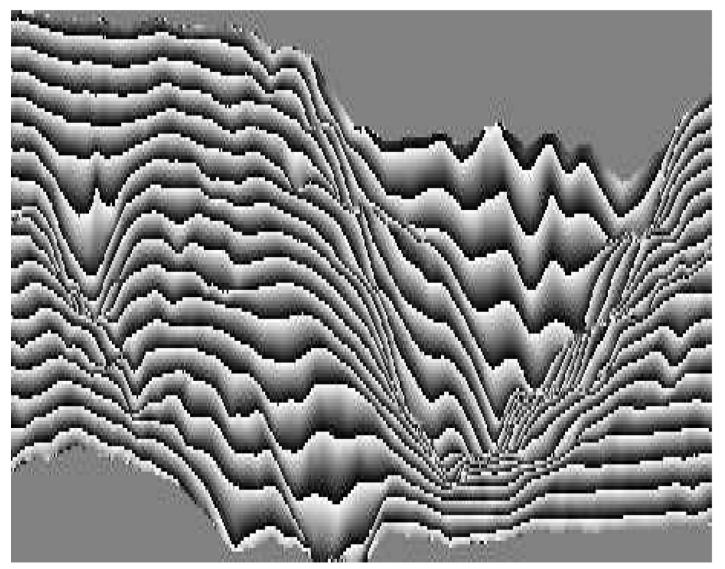

(c) Wrapped denoised Phase.

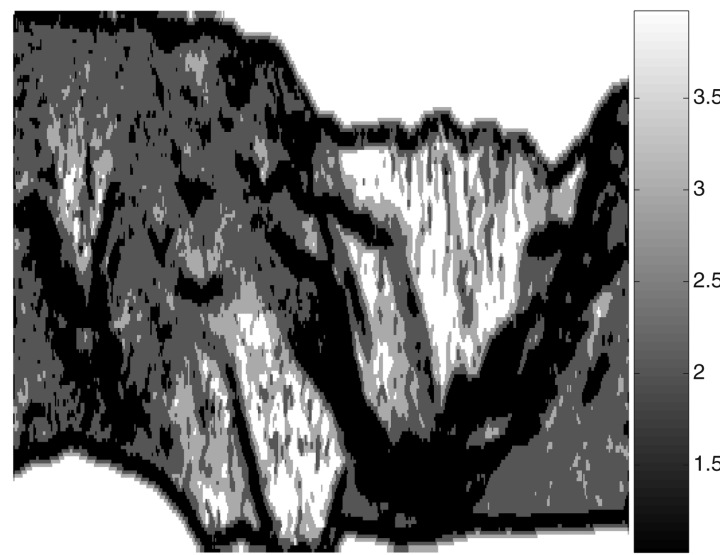

(b) Window size parameter $h$ given by ICI.

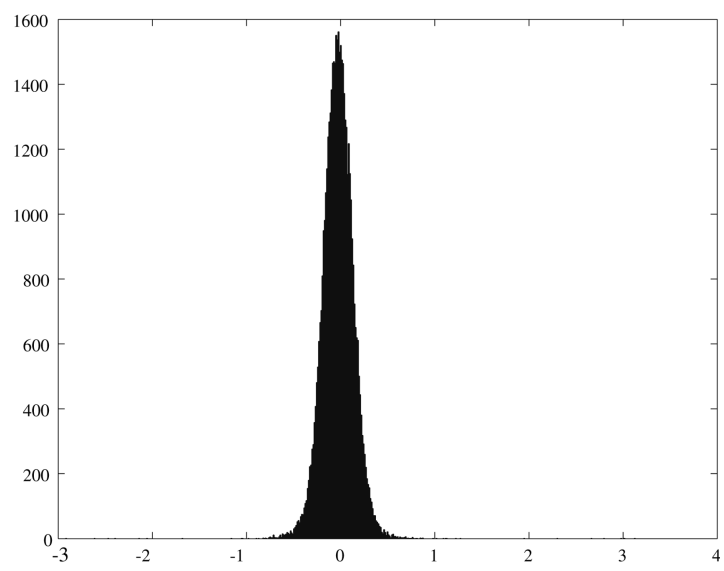

(d) Absolute phase error histogram (rad).

Fig. 7. Results obtained on a simulated SAR data based on a real surface with quality maps. Parts (a), (b), and (c) are as in Fig. 2 . The improvement in the SNR of estimate shown in part (c) is ISNR $=4 \mathrm{~dB}$. Part (d) shows the histogram of the absolute phase error in the set $X-X_{0}$. The correspondent estimation error is $\mathrm{RMSE}=0.2 \mathrm{rad}$.

extrapolated from its neighbors in the set $X-X_{0}$. More precisely, we have solved the constrained optimization problem

$$
\begin{aligned}
& \min _{\varphi_{i}, i \in X_{0}} \sum_{p \sim q}\left(\varphi_{p}-\varphi_{q}\right)^{2} \quad \text { subject to : } \varphi_{i}=\widehat{\varphi}_{i}, \\
& i \in X-X_{0}
\end{aligned}
$$

where the notation $p \sim q$ stands for first-order neighbors.

\section{Concluding Remarks}

We have introduced PEARLS, a new adaptive algorithm to denoise the wrapped phase produced by many coherent imaging systems, such as interferometric synthetic aperture radar, magnetic resonance, diffraction tomography, nondestructive testing of components, and deformation and vibration measurements.

The new methodology is based on local polynomial approximation with varying adaptive neighborhood used in reconstruction. We assume that the absolute phase is a piecewise smooth function, which is well approximated by a polynomial in a neighborhood of the estimation point. Besides the wrapped phase, also the size of this neighborhood is estimated. Thus the name PEARLS stands for phase estimation using adaptive regularization based on local smoothing.

The adaptiveness introduced by varying the size of the neighborhood used in reconstruction according to the data smoothness trades bias with variance in such a way that the window size stretches in areas where the underlying true phase is smooth and shrinks otherwise, namely in the presence of discontinuities. This is a crucial behavior for the success of any posterior phase unwrapping procedure.

To unwrap the denoised data, we apply the robust PUMA unwrapping algorithm to the denoised data. PUMA is able to deal blindly with discontinuities, as far as they have not been smoothed by the denoising step previously applied to the noisy wrapped data. The effectiveness of the overall scheme is illustrated in a series of experiments with simulated data and with data generated from a real digital elevation 


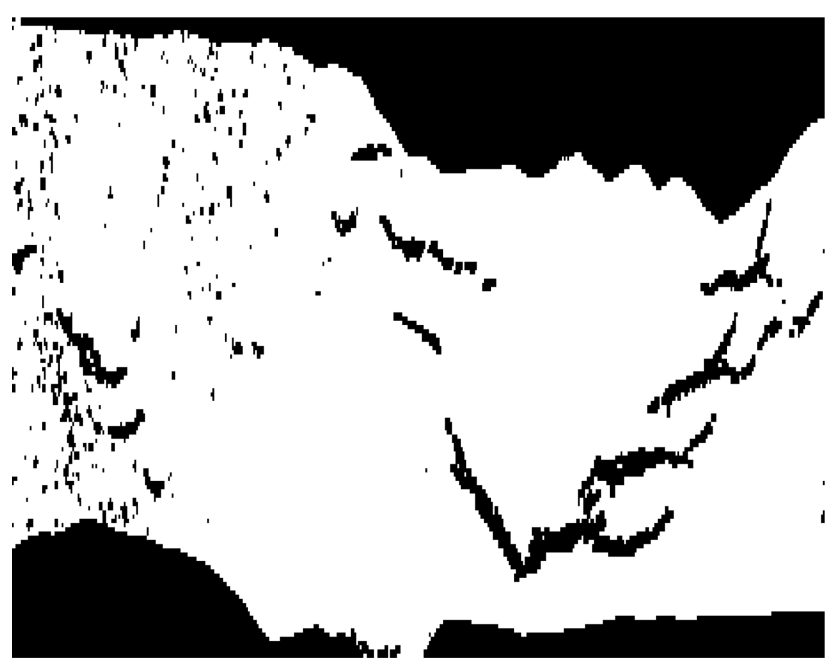

Fig. 8. Quality map relative to the interferogram shown in Fig. , part (a), computed using the phase derivative and thresholding procedures presented in [8], Chap. 3. Black color signals pixels where the interferogram is of low quality [8].

model using a high-fidelity InSAR simulator that models the SAR point spread function, the InSAR geometry, the speckle noise, and the layover and shadow phenomena. In all experiments, we obtained comparable or better performance than the stateof-the-art.

Finally, we make a brief comment on the shape of the windows used to compute the local polynomial approximation. Nonsymmetric shapes possibly yield better fittings. For example, in the neighborhood of a discontinuity, we may still use large nonsymetrical windows, assuming that the data is smooth on each side of the discontinuity. However, for computational complexity reasons, we have adopted symmetric windows. The extension of the concepts herein introduced to nonsymmetric window shapes is definitely a direction we aim to research in the future.

This research was supported by the Fundação para a Ciência e Tecnologia, under project PDCTE/CPS/ $49967 / 2003$, by the European Space Agency, under project ESA/C1:2422/2003, by the Instituto de Telecomunicações, under project IT/LA/325/2005, and by the Academy of Finland, project 213462 (Finnish Centre of Excellence program 2006-2011).

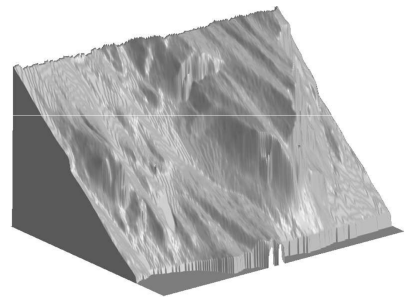

(a) True absolute phase

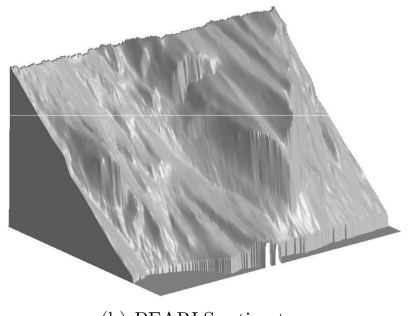

(b) PEARLS estimate.
Fig. 9. True and estimated phases. The values of the estimates phase in the set $X_{0}$ were extrapolated from the neighbors in $X-X_{0}$

\section{References}

1. L. Graham, "Synthetic interferometer radar for topographic mapping," Proc. IEEE 62, 763-768 (1974).

2. H. Zebker and R. Goldstein, "Topographic mapping from interferometric synthetic aperture radar," J. Geophys. Res. 91, 4993-4999 (1986).

3. P. Lauterbur, "Image formation by induced local interactions: examples employing nuclear magnetic resonance," Nature 242, 190-191 (1973).

4. M. Hedley and D. Rosenfeld, "A new two-dimensional phase unwrapping algorithm for MRI images," Magn. Reson. Med. 24, 177-181 (1992).

5. T. Kreis, Handbook of Holographic Interferometry: Optical and Digital Methods (Wiley-VCH, 2005).

6. A. Patil and P. Rastogi, "Moving ahead with phase," Opt. Lasers Eng. 45, 253-257 (2007).

7. K. Itoh, "Analysis of the phase unwrapping problem," Appl. Opt. 21, 2470-2470 (1982).

8. D. Ghiglia and M. Pritt, Two-Dimensional Phase Unwrapping. Theory, Algorithms, and Software (John Wiley \& Sons, 1998).

9. R. Goldstein, H. Zebker, and C. Werner, "Satellite radar interferometry: two-dimensional phase unwrapping," Radio Sci. 23, 713-720 (1988).

10. K. Stetson, J. Wahid, and P. Gauthier, "Noise-immune phase unwrapping by use of calculated wrap regions," Appl. Opt. 36, 4830-4838 (1997).

11. J. Marroquin and M. Rivera, "Quadratic regularization functionals for phase unwrapping," J. Opt. Soc. Am. 12, 2393-2400 (1995).

12. T. Flynn, "Two-dimensional phase unwrapping with minimum weighted discontinuity," J. Opt. Soc. Am. A 14, 2692-2701 (1997).

13. M. Costantini, "A novel phase unwrapping method based on network programing," IEEE Trans. Geosci. Remote Sens. 36, 813-821 (1998).

14. M. Rivera, J. Marroquin, and R. Rodriguez-Vera, "Fast algorithm for integrating inconsistent gradient fields," Appl. Opt. 36, 8381-8390 (1997).

15. J. Bioucas-Dias and J. Leitao, "The $Z \pi M$ algorithm: a method for interferometric image reconstruction in SAR/SAS," IEEE Trans. Image Process. 11, 408-422 (2002).

16. J. Bioucas-Dias and G. Valadão, "Phase unwrapping via graph cuts," IEEE Trans. Image Process. 16, 698-709 (2007).

17. Q. Kemao, "Two-dimensional windowed Fourier transform for fringe pattern analysis: principles, applications and implementations," Opt. Lasers Eng. 45, 304-317 (2007).

18. V. Katkovnik, K. Egiazarian, and J. Astola, Local Approximation Techniques in Signal and Image Processing (SPIE, 2006).

19. M. Servin, J. L. Marroquin, D. Malacara, and F. J. Cuevas, "Phase unwrapping with a regularized phase-tracking system," Appl. Opt. 37, 1917-1923 (1998).

20. Z.-P. Liang, "A model-based method for phase unwrapping," IEEE Trans. Med. Imag. 15, 893-897 (1996).

21. V. Pascazio and G. Schirinzi, "Multifrequency InSAR height reconstruction through maximum likelihood estimation of local planes parameters," IEEE Trans. Image Process. 11, 1478-1489 (2002).

22. M. Servin, F. J. Cuevas, D. Malacara, J. L. Marroguin, and R. Rodriguez-Vera, "Phase unwrapping through demodulation by use of the regularized phase-tracking technique," Appl. Opt. 38, 1934-1941 (1999).

23. M. Servin and M. Kujawinska, "Modern fringe pattern analysis in interferometry," Handbook of Optical Engineering, D. Malacara and B. J. Thompson, eds. (Dekker, 2001), Chap. 12, 373-426.

24. H. Y. Yun, C. K. Hong, and S. W. Chang, "Least-square phase estimation with multiple parameters in phase-shifting 
electronic speckle pattern interferometry," J. Opt. Soc. Am. A 20, 240-247 (2003).

25. Q. Kemao, L. T. H. Nam, L. Feng, and S. H. Soon, "Comparative analysis on some filters for wrapped phase maps," Appl. Opt. 46, 7412-7418 (2007).

26. J. Bioucas-Dias, V. Katkovnik, J. Astola, and K. Egiazarian, "Adaptive local phase approximations and global unwrap- ping," in 3DTV Conference: The True Vision - Capture, Transmission and Display of 3D Video (IEEE, 2008), pp. 253-258.

27. V. Katkovnik, J. Astola, and K. Egiazarian, "Phase local approximation (PhaseLa) technique for phase unwrap from noisy data," IEEE Trans. Image Process. 17, 833-846 (2008).

28. L. L. Scharf, Statistical Signal Processing, Detection Estimation and Time Series Analysis (Addison-Wesley, 1991). 